\title{
Casimir quantum levitation tuned by means of material properties and geometries
}

\author{
Maofeng Dou, ${ }^{1, *}$ Fei Lou, ${ }^{2}$ Mathias Boström, ${ }^{1,3,4}$ Iver Brevik, ${ }^{3}$ and Clas Persson ${ }^{1,4,5}$ \\ ${ }^{1}$ Department of Materials Science and Engineering, Royal Institute of Technology, SE-100 44 Stockholm, Sweden \\ ${ }^{2}$ Laboratory of Photonics and Microwave Engineering, KTH-Royal Institute of Technology, SE-164 40 Kista, Sweden \\ ${ }^{3}$ Department of Energy and Process Engineering, Norwegian University of Science and Technology, NO-7491 Trondheim, Norway \\ ${ }^{4}$ Centre for Material Science and Nanotechnology, University of Oslo, P.O. Box 1048, Blindern, NO-0316 Oslo, Norway \\ ${ }^{5}$ Department of Physics, University of Oslo, P.O. Box 1048, Blindern, NO-0316 Oslo, Norway \\ (Received 25 February 2014; revised manuscript received 13 May 2014; published 23 May 2014)
}

\begin{abstract}
The Casimir force between two surfaces is attractive in most cases. Although stable suspension of nano-objects has been achieved, the sophisticated geometries make them difficult to be merged with well-established thin film processes. We find that by introducing thin film surface coating on porous substrates, a repulsive to attractive force transition is achieved when the separations are increased in planar geometries, resulting in a stable suspension of two surfaces near the force transition separation. Both the magnitude of the force and the transition distance can be flexibly tailored though modifying the properties of the considered materials, that is, thin film thickness, doping concentration, and porosity. This stable suspension can be used to design new nanodevices with ultralow friction. Moreover, it might be convenient to merge this thin film coating approach with micro- and nanofabrication processes in the future.
\end{abstract}

DOI: 10.1103/PhysRevB.89.201407

PACS number(s): 42.50.Lc, 42.15.Fr, 77.22.Ch, 34.20.Cf

If the distance of two neutral objects is very close (typically nanoscale), the Casimir force, which arises from quantum fluctuations, becomes dominant and increases rapidly as the distance decreases. The force is generally attractive in configurations with vacuum separated dielectrics or metallic objects. By properly choosing the materials, such as magnetic compounds [1], fluid-separated dielectrics [2], or topological insulators [3], the force can, however, turn out to be repulsive. Although the fundamental theory of Casimir force has been well studied, tuning this force for potential technology applications is still in its infancy and has recently attracted considerable attention. As microelectromechanical systems $[4,5]$ evolve into nanoelectromechanical systems, the attractive Casimir stiction phenomenon becomes important and has to be faced. On the other hand, such phenomena may also be of significant interest when designing new nanodevices, such as the Casimir anharmonic oscillator [6,7] or the ultrasensitive sensor [8]. Several interesting works have appeared regarding the use of repulsive Casimir force to achieve stable levitation of nano-objects, such as cylinder in cylinder [9], sphere on surface [10], or rotated hockey pucks [11]. Most of them are, however, achieved in sophisticated geometries that are difficult to fabricate using well-established micro- and nanofabrication technologies. In this work, we demonstrate by means of multilayer modeling that the Casimir induced quantum levitation can be achieved in traditional planar geometry containing nanosheets. Moreover, this configuration could be further extended to thin film cladding on a mesoporous substrate without destroying the quantum levitation. Remarkably, both the magnitude of the force and the levitation distance are tunable through modifying the properties of the involved materials, that is, the thickness of the film, the doping concentration, and the porosity.

In this Rapid Communication we consider a configuration of four layers, labeled as $m, j, s$, and $l$. The retarded Casimir force acting on layer $j$ per unit area includes the contributions from both the transverse magnetic (TM) and transverse electric
(TE) modes. It can be written as a sum over discrete imaginary frequencies $\omega_{n}[12,13]$ :

$$
\begin{aligned}
F & =\sum_{n=0}^{\infty}{ }^{\prime} g\left(\omega_{n}\right) \\
& =\sum_{n=0}^{\infty}, \frac{k_{B} T}{\pi} \int_{0}^{\infty} d k_{\perp} k_{\perp} \kappa_{j} \sum_{q=\mathrm{TM}}^{\mathrm{TE}} \frac{r_{j-}^{q} r_{j+}^{q} e^{-2 \kappa_{j} d_{j}}}{1-r_{j-}^{q} r_{j+}^{q} e^{-2 \kappa_{j} d_{j}}},
\end{aligned}
$$

where $d_{j}$ is the width of the layer $j, a$ is the thickness of the nanosheet layer $s, r_{j-}^{q}$ and $r_{j+}^{q}$ are the reflectivity coefficients on the left and right sides of layer $j$, respectively, and $\kappa_{j}=\sqrt{k_{\perp}^{2}-\varepsilon_{j}\left(i \omega_{n}\right) \mu_{j}\left(i \omega_{n}\right)\left(i \omega_{n} / c\right)^{2}}$, where $\varepsilon_{j}$ and $\mu_{j}$ are the dielectric and magnetic "constants" of the material $j$, and $g\left(\omega_{n}\right)$ is the spectral function that is frequency decomposition of the Casimir force. The prime on the summation indicates that the $n=0$ term shall be divided by 2 . The expressions for the reflectivity coefficients $r_{j-}^{q}$ and $r_{j-}^{q}$ are

$$
\begin{aligned}
& r_{j-}^{q}=r_{m j}^{q}=\frac{\kappa_{m}-\gamma_{m j}^{q} \kappa_{j}}{\kappa_{m}+\gamma_{m j}^{q} \kappa_{j}}, \\
& r_{j+}^{q}=r_{j s l}^{q}=\frac{r_{j s}^{q}+r_{s l}^{q} e^{-2 \kappa_{s} a_{s}}}{1+r_{j s}^{q} r_{s l}^{q} e^{-2 \kappa_{s} a_{s}}},
\end{aligned}
$$

where $r_{j s}^{q}$ and $r_{s l}^{q}$ have the similar expression as $r_{m j}^{q}, \gamma_{i j}^{q}=$ $\mu_{i} / \mu_{j}$ for $q=\mathrm{TE}$ and $\gamma_{i j}^{q}=\varepsilon_{i} / \varepsilon_{j}$ for $q=\mathrm{TM}$. In the nonretarded limit, the speed of light $c$ goes to infinite and $\kappa_{j} \rightarrow k_{\perp}$, which is a valid approximation for short separations. For the nonmagnetic materials in our case, the reflectivity coefficients $r_{j-}$ and $r_{j+}$ for TE modes go to the limit of zero. The contributions from TE modes are extremely small compared to TM contributions.

The complex dielectric functions can be determined employing a first-principles approach involving density functional theory. The electronic structure neglecting electronphonon coupling is then obtained from the partial self- 
consistent $\mathrm{GW}_{0}$ method where the Green's functions are updated iteratively, whereas the screened Coulomb potential $W$ is fixed $[14,15]$. The imaginary part of the dielectric function is calculated in the long wavelength limit from the joint density of states and the optical momentum matrix. Since the dielectric function in polar materials depends strongly on the electron-phonon coupling, we model this contribution to the dielectric function using the Lorentz model and the Kramers-Heisenberg formula with a multiphonon contribution $[16,17]$, where the phonon frequencies are determined via the linear response method. Calculated dielectric functions of $\mathrm{SnO}_{2}$ on the imaginary frequency axis with different doping concentrations, together with measured values for Teflon [18] and cyclodecane [19], are shown in Fig. 1.

Conceptually, the Casimir force between plates 1 and 2 separated by medium 3 depends on the dielectric properties. The force is repulsive if the dielectric functions $\varepsilon_{1}, \varepsilon_{2}$, and $\varepsilon_{3}$ on the imaginary frequency satisfy $\varepsilon_{1}<\varepsilon_{3}<\varepsilon_{2}$, whereas it is attractive otherwise. The behavior is complicated if the above relation is satisfied at some frequencies and violated at others and/or if the system contains finite-size configurations. For the Teflon-cyclodecane- $\mathrm{SnO}_{2}$ semi-infinite configuration, the force is repulsive at all separations (Fig. 2). Replacing the $\mathrm{SnO}_{2}$ semi-infinite with a nanosheet, however, the force turns attractive at large separations and remains repulsive at small separations, resulting in a stable separation distance $d_{c}$ at which the Casimir force acting on the cyclodecane film is zero. This distance $d_{c}$ depends strongly on the thickness of the $\mathrm{SnO}_{2}$ nanosheet, for instance, $d_{c} \sim 50$ and $10 \mathrm{~nm}$ for thicknesses $a \sim 10$ and $3 \mathrm{~nm}$, respectively. For the Teflon-cyclodecane-air configuration, the force is attractive at all separations. It should be pointed out that it is not solely the finite thickness that gives rise to the repulsive-attractive force transition in the configuration with the nanosheet. As shown in Fig. 3(a), the nonretarded Casmir force in the nanosheet configuration is

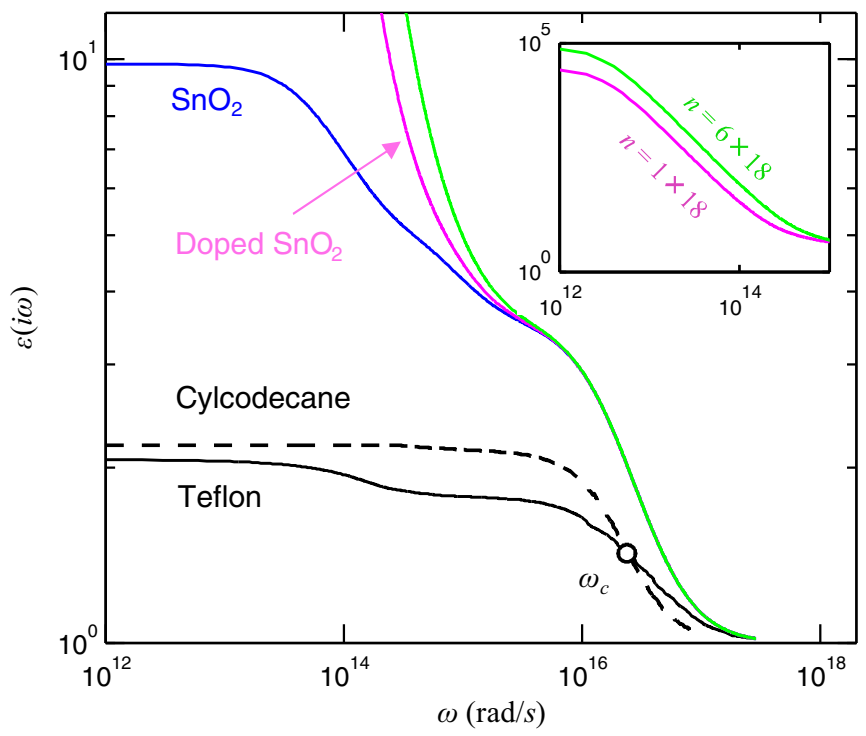

FIG. 1. (Color online) The calculated dielectric functions along the imaginary frequency axis for rutile $\mathrm{SnO}_{2}$ with doping concentration $n=1 \times 10^{-18}$ and $n=6 \times 10^{-18} \mathrm{~cm}^{-3}$, respectively. The data of cyclodecane and Teflon are from Refs. [15] and [16], respectively.

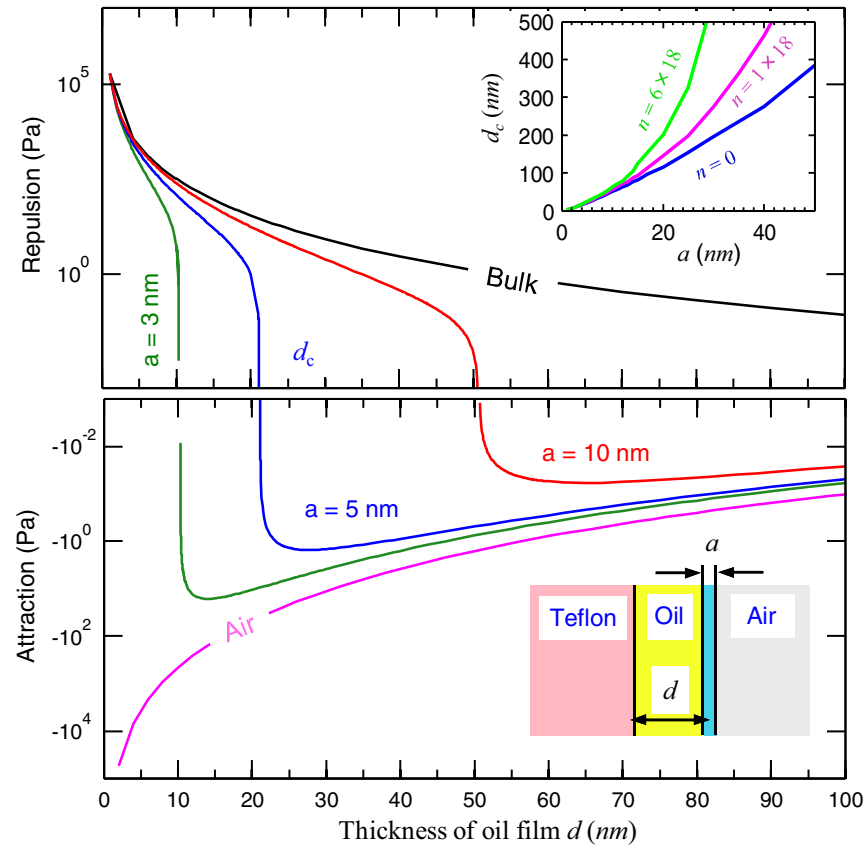

FIG. 2. (Color online) The Casimir-Lifshitz force between Teflon and $\mathrm{SnO}_{2}$ nanosheet with different thickness in cyclodecane at $300 \mathrm{~K}$ (schematically shown in inset, bottom). The forces between Teflon and $\mathrm{SnO}_{2}$ in cyclodecane as well as cyclodecane on the Teflon surface are presented for comparison. Inset (upper): The stable separation $d_{c}$ versus the thickness of the $\mathrm{SnO}_{2}$ nanosheet with doping concentration $n=0.0, n=1 \times 10^{-18}$, and $n=6 \times 10^{-18} \mathrm{~cm}^{-3}$, respectively.

attractive at all separations but the corresponding retarded force shows a change of sign at a separation of about $21 \mathrm{~nm}$. For the Teflon-cyclodecane- $\mathrm{SnO}_{2}$ semi-infinite configuration, however, both the retarded and nonretarded forces are repulsive at all separations. This indicates that the combination of the finite thickness and the retardation contributes to the repulsive-attractive force transition. The spectral function $g\left(\omega_{n}\right)$ corresponds to a repulsive force at separation $d=$ $25 \mathrm{~nm}$ for Teflon-cyclodecane- $\mathrm{SnO}_{2}$ but to an attractive force for a nanosheet. For the nanosheet with thickness far below the skin depth, the material becomes more transparent and consequently the electromagnetic modes confined in the optical cavity (cyclodecane layer) have significantly reduced influence compared to that of $\mathrm{SnO}_{2}$ surface configuration. The total force becomes attractive. The retardation effect, however, turns the attractive $g\left(\omega_{n}\right)$ at low and high frequencies into a repulsive one and remains attractive at the intermediate frequencies, i.e., the frequencies between $\sim 6.7 \times 10^{14}$ and $8.2 \times 10^{15} \mathrm{rad} / \mathrm{s}$ [see Fig. 3(b)]. As the separation decreases, the repulsive contribution dominates and the total force becomes repulsive at small separation. The distance $d_{c}$ can also be tuned through modifying the dielectric response of the materials. Rutile $\mathrm{SnO}_{2}$ is a wide-gap semiconductor with large band curvature at the bottom of the conduction band [20]. The dielectric function at low frequencies can be modified through electron doping. The plasma of free carriers in the doped nanosheets increases the dielectric function at frequencies in the infrared range. Consequently, the repulsive contribution 

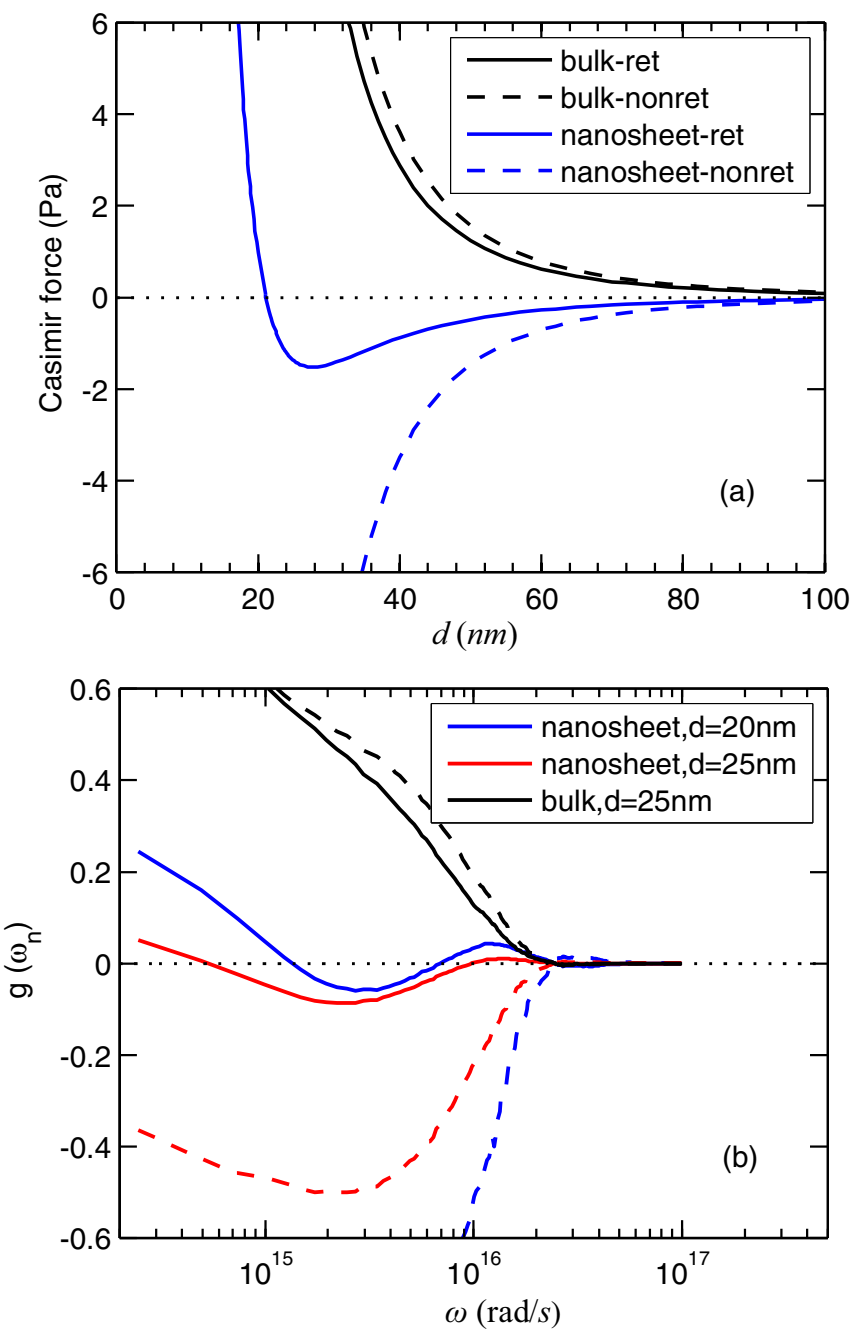

FIG. 3. (Color online) (a) The retarded (solid line) and nonretarded (dashed line) Casimir-Lifshitz force between Teflon and $\mathrm{SnO}_{2}$ surface, Teflon and $\mathrm{SnO}_{2}$ nanosheet separated by cyclodecane. The thickness of the nanosheet is $5 \mathrm{~nm}$. (b) The corresponding spectral functions $g\left(\omega_{n}\right)$ at different separations.

becomes enhanced compared to the case of no doping and the equilibrium separation $d_{c}$ increases with increase of the doping concentration (inset of Fig. 2). So far, gravitational effects have been omitted. They do not play a role when the layers are vertically oriented. However, if the layers are placed horizontally, one may have to take into account the gravitational potential (see the Supplemental Material [21]).

Although the configuration with a nanosheet presents repulsive-attractive force transition and can stably suspend the nanosheet, it is difficult to manipulate systems with a single nanosheet without any substrate in the process of fabrication. An alternative way is to use ultrathin coating by replacing the air cladding on top of the $\mathrm{SnO}_{2}$ nanosheet with a mesoporous material. The mesoporous material consists of host medium and nanopores where its dielectric function can be tuned through modifying the pores structures. To investigate this concept, a hexagonal mesoporous silica film (MCM-41) can be taken as a typical example. The dielectric function is calculated using the effective medium approximation (EMA), wherein the material is treated as a homogeneous medium. The validity of the EMA approach can be confirmed through comparison with numerical calculations [22]. It is found that if the film thickness is significantly larger than the pore size (as in the case in our work) the effective medium models are fairly accurate. For the TE polarized wave, EMA described by the volume averaging theory (VAT) matches accurately with numerical results, while for the TM polarized light the parallel model [23] gives the best predictions. Mathematically, the effective dielectric function for the VAT models is $\varepsilon_{\text {eff }}=(1-\phi) \varepsilon_{c}+\phi \varepsilon_{d}$, where $\phi$ is the porosity and $\varepsilon_{c}$ and $\varepsilon_{d}$ are the dielectric functions of the continuous (silica) and the dispersed (air) phases, respectively. In the studied hexagonal mesoporous material, with nanopore diameter $D$ and lattice parameter $a_{0}$, the porosity is expressed as $\phi=\pi \sqrt{3} D^{2} / 6 a_{0}^{2}$ [23].

To analyze the Casimir force in porous materials, we choose two typical mesoporous silica materials that have been experimentally synthesized [24] to replace the air. Both mesoporous materials consist of hexagonal ordered cylindrical pores with pore diameters of 8.5 and $4.7 \mathrm{~nm}$, respectively. The lattice parameters are about 12.0 and $5.4 \mathrm{~nm}$, respectively, and the corresponding porosities are $45 \%$ and $68 \%$. For the mesoporous silica with a porosity of $68 \%$, the Casimir force is repulsive (attractive) at the separation below (above) $19 \mathrm{~nm}$. This distance increases to $37 \mathrm{~nm}$ as the porosity of mesoporous materials decreases to $45 \%$ (inset of Fig. 4). When the porosity is smaller than $30 \%$, the force becomes repulsive at all separations. Not only the distance $d_{c}$ and the force change sign, but also the magnitude of the Casimir force can be tuned through different combinations of the

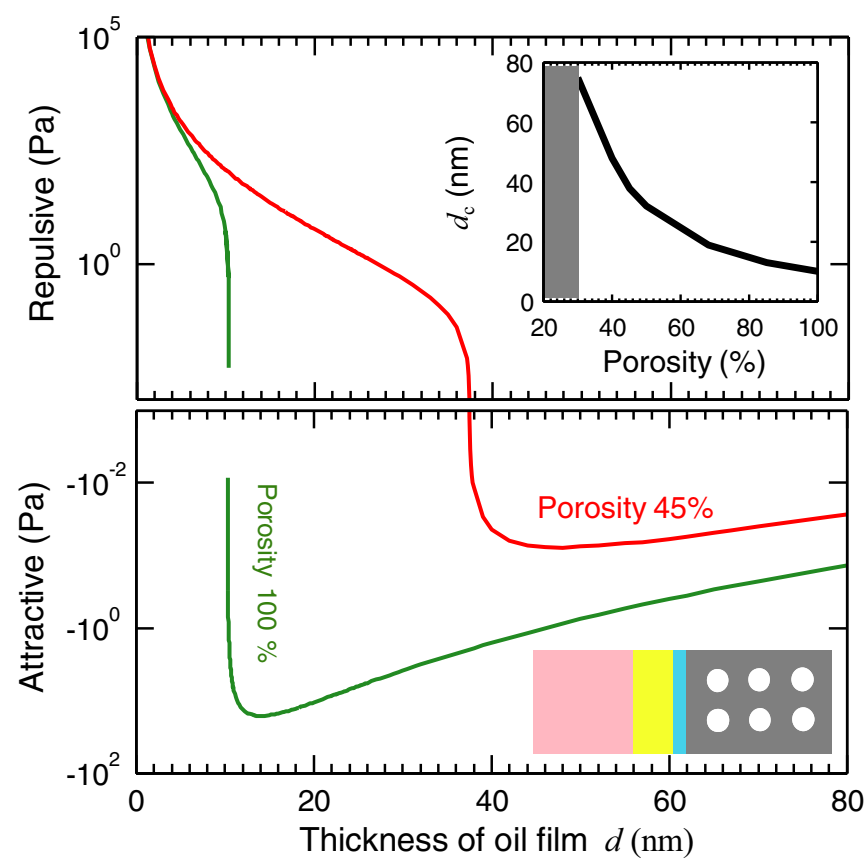

FIG. 4. (Color online) The Casimir-Lifshitz force between Teflon and $\mathrm{SnO}_{2}$ thin film coated mesoporous silica in cyclodecane at $300 \mathrm{~K}$. The porosity of the mesoporous silica is $45 \%$ and $68 \%$, respectively. Inset: The dependence of the stable separation $d_{\mathrm{c}}$ on the porosity of the mesoporous silica. 
interacting materials, through thin film thickness, doping concentration, and porosity. This large number of tunable factors implies a flexibility to tailor the Casimir force for different application requirements. The results discussed in our Rapid Communication, showing the great versatility in the magnitude of the Casimir force, ought to be of definite interest in dealing with the stiction problem (see Supplemental Material [21]).

In conclusion, we demonstrated that finite thickness and retardation in conjunction can turn the Casimir force between Teflon and the rutile $\mathrm{SnO}_{2}$ nanosheet immersed in cyclodecane from repulsive to attractive at large separations but remains repulsive at small separations, resulting in a stable equilibrium distance where the total force is zero. To overcome the difficulties of manipulating nanosheets in a fabrication process, the system is extended to surface coating on porous substrates that can be merged with well-established thin film processes. Moreover, this stable quantum-levitation equilibrium state can be used to design new types of nanodevices. Thus the results and conclusion in this work present a guideline for designing Casimir force through modifying the material properties. The force sign, magnitude, and the distance, can be tuned from the characteristic properties of the materials involved, that is, film thickness, doping concentration, and porosity. The exact levitation distance may vary with measurement: This is partly due to the dielectric functions for the nanosheet and for the thin film being assumed to be equal to that of the bulk materials, and partly due to the surface roughness not taken into account in the Casimir force calculation.

This work is financially supported by the ESF exchange grant within the activity "New Trends and Applications of the Casimir Effect" through the network Casimir, the China Scholarship Council, the Swedish Research Council (Contract No. C0485101), and the Research Council of Norway (Contract No. 221469). We acknowledge access to HPC resources at NSC through SNIC/SNAC and at USIT through NORTUR. M.D. thanks Simen Å. Ellingsen for fruitful discussions.
[1] N. Inui, J. Appl. Phys. 111, 074304 (2012).

[2] J. N. Munday, F. Capasso, and V. A. Parsegian, Nature (London) 457, 170 (2009).

[3] A. G. Grushin and A. Cortijo, Phys. Rev. Lett. 106, 020403 (2011).

[4] F. M. Serry, D. Walliser, and G. J. Maclay, J. Appl. Phys. 84, 2501 (1998)

[5] B. Bhushan, J. Vac. Sci. Technol. B 21, 2262 (2003).

[6] F. M. Serry, D. Walliser, and G. J. Maclay, J. Microelectormech. Syst. 4, 193 (1995).

[7] H. B. Chan, V. A. Aksyuk, R. N. Kleiman, D. J. Bishop, and F. Capasso, Phys. Rev. Lett. 87, 211801 (2001).

[8] E. L. Carter, M. Ward, and C. Anthony, IEEE Sensors Proceeding (IEEE, Christchurch, 2009), p. 229.

[9] S. J. Rahi and S. Zaheer, Phys. Rev. Lett. 104, 070405 (2010).

[10] A. W. Rodriguez, A. P. McCauley, D. Woolf, F. Capasso, J. D. Joannopoulos, and S. G. Johnson, Phys. Rev. Lett. 104, 160402 (2010).

[11] M. Levin, A. P. McCauley, A. W. Rodriguez, M. T. Homer Reid, and S. G. Johnson, Phys. Rev. Lett. 105, 090403 (2010).
[12] M. S. Tomaš, Phys. Rev. A 66, 052103 (2002).

[13] S. A. Ellingsen, J. Phys. A: Math. Theor. 40, 1951 (2007).

[14] M. Shishkin and G. Kresse, Phys. Rev. B 75, 235102 (2007).

[15] M. Dou and C. Persson, Phys. Status Solidi A 209, 75 (2012).

[16] C. Klingshirn, Semiconductor Optics (Springer, Berlin, 2004).

[17] H. Kuzmany, Solid State Spectroscopy: An Introduction (Springer, Berlin, 2009)

[18] P. J. van Zwol and G. Palasantzas, Phys. Rev. A 81, 062502 (2010).

[19] T. Masuda, Y. Matsuki, and T. Shimoda, J. Colloid Interface Sci. 340, 298 (2009).

[20] M. Dou and C. Persson, J. Appl. Phys. 113, 083703 (2013).

[21] See Supplemental Material at http://link.aps.org/supplemental/ 10.1103/PhysRevB.89.201407 for details regarding the gravitational effects and Casimir friction and stiction.

[22] M. M. Braun and L. Pilon, Thin Solid Films 496, 505 (2006).

[23] A. Navid and L. Pilon, Thin Solid Films 516, 4159 (2008).

[24] M. Dou, T. Yu, S. Jin, and C. Persson, Sensor Lett. 11, 1530 (2013). 